\title{
Influence of the main neutron absorbers poisons coupled to the Point Kinetics model by the Rosenbrock's method
}

\author{
Schaun $^{\mathrm{a}}$ N. B., Tumelero ${ }^{\mathrm{a}}$ F., Petersen ${ }^{\mathrm{a}}$ C. Z. \\ ${ }^{a}$ Federal University of Pelotas, Campus Capão do Leão, Graduate Program in Mathematical Modeling \\ Postal code 354 - CEP 96001 - 970, Capão do Leão - RS, Brazil. \\ natalia.schaun@ufpel.edu.br
}

\begin{abstract}
In this work, the Neutron Point Kinetics equations are solved for six groups of delayed neutron precursors and different types of ramp reactivity, considering the temperature effects by the Rosenbrock's method, to verify the methodology. Furthermore, the classical model is solved by inserting the effects of the main neutron poisons, considering constant reactivity for a group of precursors. The simulation consists of inserting a negative constant reactivity, simulating a reactor in its shutdown phase. Then, positive constant reactivity is inserted, simulating power resumption in a reactor already poisoned, to analyzing the final behavior of the neutron density. The simulation achieved its goal of simulating the behavior of the neutron poisons, so that the graphs make physical sense as expected. Therefore, it was found that the proposed method overcame the stiffness of the Neutron Point Kinetics model, and also solved a nonlinear problem by the inclusion of temperature and neutron poisons in the system.
\end{abstract}

Keywords in english: Rosenbrock's Method, Neutron Point Kinetics, Temperature feedback, Neutron poisons. 


\section{INTRODUCTION}

The safety of a nuclear power plant is an extremely relevant topic in reactor physics. In a plant, more specifically inside the reactor core, there must be an equilibrium in the neutron balance, referring to the loss and gain of neutrons, ensuring control in the chain reaction for energy generation.It is in this sense that researchers in the area have invested in studies through models and mathematical methods that describe accurately, quickly and reliably the physical situation of the problem, important for decision making and operational control of a nuclear power plant. athematical models in nuclear reactor physics must take into account all aspects, variables and possible modifications in the reactor structure by internal and external agents, influencing the nuclear reaction and, consequently, the final analysis in the overall reactor behavior.

The most complete physico-mathematical model, when time-dependent and considering delayed neutrons [1], which determines the neutron population is known as the Neutron Transport equation. However, this model becomes impossible to solve when considering in its composition angular, energy, spatial and temporal variation in the equations. However, simpler models can be obtained from coherent physical simplifications of this equation. One of the commonly used approximations is to treat the transport equation as a diffusive process, known as the neutron diffusion equation [2]. Even so, according to the literature, other models have been ensuring the simulation of the transient behavior of nuclear reactors, important for reactor control. These models can be obtained through the Kinetics equations, which can be divided into the equations of the Neutron Point Kinetics (NPKE) and the equations of the Neutron Spatial Kinetics (NSKE). The Neutron Space Kinetics equations consider both the variation of the flux with time and its spatial deformity, while the Point Neutron Kinetics equations are exclusively interested in the variation of the flux amplitude with time, making these equations exclusively time-dependent. In this work, the model employed is that of Neutron Point Kinetics considering temperature effects and, subsequently, neutron absorbers poisons.

The Neutron Point Kinetics equations form a system of coupled ordinary differential equations (ODEs) that describe the neutron density and the concentration of delayed neutron precursors [3]. 
Although a relatively simple model, it plays a very important role in reactor physics as it can be used, when solved properly, for near real-time prediction of the reactor power transient [3], important for reactor decision making and control.

The effects of temperature have an important influence on the operation of a reactor and the safety of the system. The insertion of temperature feedback causes relevant changes in the neutron flux that, consequently, according to Silva [4], alter the power of the reactor. The same happens with the presence of fission product poisons in the reactor core. The poisons xenon-135 and samarium-149 have high neutron absorption cross-sections that can delay the reactor response time for the safe resumption of power.

The accident at Chernobyl, for example, had as one of the causes of its occurrence the neglect of the effects of core poisoning during a safety test. The positive reactivity inserted was not able to overcome the negative reactivity arising from the absorbers poisons, causing the reactor to shut down. As a result, numerous test procedures and safety regulations were violated in an attempt to compensate for the negative reactivity caused by the poisoning in the core. Due to the irregular procedures taken, various thermal-hydraulic problems began to appear in the system, leading to a series of events leading up to the accident.

In the literature some works address the model of the Neutron Point Kinetics coupled with temperature effects, such as Tashakor et al. [5] that develop a numerical solution, taking into account, besides the temperature feedback, the fuel burning, using only one group of delayed neutron precursors. In Silva [4] the decomposition method presented in Petersen [6] is used to obtain an analytical solution of the problem, where the nonlinearity is treated using Adomian polynomials. Tumelero [7] presents a solution of the equations of the Neutron Point Kinetics applying the polynomial approximation method, also considering the temperature effects.

In the literature, there are also works involving the effects of poisons in the nuclear reactor. We point out that simulations with the coupling of the effects of the main neutron absorbers poisons to the Point Kinetic Model are not very common in the literature. Few works bring these simulations, to verify the validity of the methodologies and the physical meaning of the results. Among them, we can mention that of Espinosa [8] that works with the Neutron Point Kinetic equations taking into account the effects of xenon-135 and samarium-149 poisons, in which the new system of equations is solved through a decomposition method, which expands the nonlinear terms in an infinite series 
obtaining a recursive system, treating the nonlinearity by Adomian polynomials. Recently, Paganim et al. [9] solve the NPKEs considering not only the effects of the main neutron poisons, but also the effects of some transuranics by the same method of Espinosa [8], obtaining results consistent with the expected ones.

The Neutron Point Kinetics equations have a fundamental characteristic due to the difference in the lifetimes of prompt and delayed neutrons, called stiffness. With this, numerical methods that could overcome it were sought in the literature. The Rosenbrock's method (ROS) has been shown in the literature to be satisfactory in the solution of stiff problems. Galina [10] presents numerical solutions for stiff radioactive decay chain problems involving natural and artificial chains through the Rosenbrock's method, producing consistent results. Recently, in chemical kinetics, Sehnem [11] uses the method to solve a stiff system of ordinary differential equations to moderate the degree of stiffness and decrease the number of species involved in the combustion of the element methane. In the work of Yang and Jevremovic [12], the Rosenbrock's method is used to solve the Neutrons Point Kinetics equations itself with different types of reactivity insertions, in which the results are compared with reference data in the literature, considered a benchmark in reactor physics.

In this work, the goal is to highlight the importance of considering in the model the effects of both temperature and fission product poisons in the final behavior of the neutron density. For this, the equations of the Neutron Point Kinetics are solved considering the temperature effects, to verify the methodology, by the Rosenbrock's method of fourth-order and four stages, comparing with the EPCA method of Picca et al. [13]. Subsequently, the effects of the main neutron absorbers poisons are coupled to the model, simulating two cases of reactivity: negative and positive constant.

\section{MATERIALS AND METHODS}

\subsection{The Model with Temperature Feedback}

The reactor is assumed to have a negative temperature coefficient $(\alpha)$. That is, when a small perturbation in reactivity is inserted, considering temperature feedback, the actual reactivity of the reactor is 


$$
\rho=\rho_{0}-\alpha\left(T-T_{0}\right)
$$

where $\rho_{0}$ is a constant and $T_{0}$ is the initial temperature of the system.

After the reactivity be inserted into the reactor the adiabatic model is still employed, so, has been:

$$
\frac{d T}{d t}=H n(t)
$$

where $H$ is the reciprocal of the reactor heat capacity. Combining (1) and (2), one has:

$$
\frac{d \rho}{d t}=-\alpha H n(t)
$$

Renaming $B=\alpha H$, results in:

$$
\frac{d \rho}{d t}=-B n(t)
$$

where $B$ is called the "shutdown coefficient".

Thus, considering the Point Kinetics equations and the Newtonian feedback model, the following system of ordinary differential equations is obtained:

$$
\begin{aligned}
& \frac{d n(t)}{d t}=\frac{(\rho(t, n)-B)}{\Lambda} n(t)+\sum_{i=1}^{6} \lambda_{i} C_{i}(t) \\
& \frac{d C_{i}(t)}{d t}=\frac{\beta_{i}}{\Lambda} n(t)-\lambda_{i} C_{i}(t) \\
& \frac{d \rho(t, n)}{d t}=\frac{d \rho_{0}}{d t}-B n(t)
\end{aligned},
$$

for $i=1,2, \ldots, 6$, where $n(t)$ given in $\left[\mathrm{cm}^{-3}\right]$ is the neutron density in time $t, \rho(t, n)$ is the time and density dependent reactivity, $\beta$ the total fraction of delayed neutrons, $\Lambda$ given in $[s]$ is the mean generation time between neutron birth and subsequent collision, $\lambda_{i}$ given in $\left[s^{-1}\right]$ are the radioactive decay constants for each group $i$ of precursors, $C_{i}(t)$ given in $\left[\mathrm{cm}^{-3}\right]$ are the delayed neutron precursor concentrations for each group $i$ at time $t$.

Consider the following initial conditions for the system described in Equation (5): 


$$
\begin{aligned}
& n(0)=n_{0} \\
& C_{i}(0)=\frac{\beta_{i}}{\lambda_{i} \Lambda}, \\
& \rho(0)=\rho_{0}
\end{aligned}
$$

for $i=1,2, \ldots, 6$. Thus, rewriting Equation (5) in matrix form, we have:

$$
\frac{d \mathbf{y}(t)}{d t}=\mathbf{A}(t, n(t)) \mathbf{y}(t)+\mathbf{g}(t)
$$

where

$$
\mathbf{y}(t)=\left(\begin{array}{c}
n(t) \\
C_{1}(t) \\
\vdots \\
C_{6}(t) \\
\rho(t)
\end{array}\right), \mathbf{A}(t, n(t))=\left(\begin{array}{cccccc}
\frac{\rho(t, n(t))-\beta}{\Lambda} & \lambda_{1} & \lambda_{2} & \cdots & \lambda_{6} & 0 \\
\frac{\beta_{1}}{\Lambda} & -\lambda_{1} & 0 & \cdots & \ldots & 0 \\
\frac{\beta_{2}}{\Lambda} & 0 & -\lambda_{2} & & & \ldots \\
\cdots & \ldots & & \ldots & & \ldots \\
\frac{\beta_{6}}{\Lambda} & 0 & \ldots & 0 & -\lambda_{6} & 0 \\
-B & 0 & \ldots & & \ldots & 0
\end{array}\right) \text { and } \mathbf{g}(t)=\left(\begin{array}{c}
0 \\
0 \\
\vdots \\
0 \\
\frac{d \rho_{0}}{d t}
\end{array}\right)(8)
$$

\subsection{The Model with the Effects of Neutron Absorbers Poisons}

The Neutron Point Kinetics model coupled with the effects of the fission product poisons is presented in Equation (9). Due to the effects of the poisons xenon-135 and samarium-149 have high neutron absorption cross-sections, it is relevant to include two loss terms in the density variation equation. Thus, we have the following system of equations 


$$
\begin{aligned}
& \frac{d n(t)}{d t}=\frac{\rho(t)-\beta}{\Lambda} n(t)+\lambda C(t)-\sigma_{a, X e} C_{X e}(t) v n(t)-\sigma_{a, S m} C_{S m}(t) v n(t) \\
& \frac{d C(t)}{d t}=\frac{\beta}{\Lambda} n(t)-\lambda C(t) \\
& \frac{d C_{I}(t)}{d t}=\gamma_{I} \sum_{f} v n(t)-\lambda_{I} C_{I}(t) \\
& \frac{d C_{X e}(t)}{d t}=\gamma_{X e} \sum_{f} v n(t)+\lambda_{I} C_{I}(t)-\lambda_{X e} C_{X e}(t)-\sigma_{a, X e} C_{X e}(t) v n(t) \\
& \frac{d C_{P m}(t)}{d t}=\gamma_{P m} \sum_{f} v n(t)-\lambda_{P m} C_{P m}(t) \\
& \frac{d C_{S m}(t)}{d t}=\lambda_{P m} C_{P m}(t)-\sigma_{a, S m} C_{S m}(t) v n(t)
\end{aligned}
$$

where $v$ given in $[\mathrm{cm} / \mathrm{h}]$ is the velocity of the neutron, $\sum_{f}$ given in $\left[\mathrm{cm}^{-1}\right]$ is the macroscopic cross-section of fission, $\lambda$ given in $\left[h^{-1}\right]$ is the delayed neutron precursor decay constant, $C(t)$ given in $\left[\mathrm{cm}^{-3}\right]$ is the concentration of delayed neutron precursors at time $t, \sigma_{a, X e}$ given in $\left[\mathrm{cm}^{2}\right]$ is the microscopic cross-section of absorption of the element xenon-135, $C_{X e}(t)$ given in $\left[\mathrm{cm}^{-3}\right]$ is the concentration of xenon-135 in time $t, \sigma_{a, S m}$ given in $\left[\mathrm{cm}^{2}\right]$ is the microscopic cross-section of absorption of samarium-149, $C_{S m}(t)$ given in $\left[\mathrm{cm}^{-3}\right]$ is the concentration of samarium-149 in time $t, \gamma_{I}$ is the fission yield of the nuclide iodine-135, $\lambda_{I}$ given in $\left[h^{-1}\right]$ is the radioactive decay constant of iodine-135, $C_{I}(t)$ given in $\left[\mathrm{cm}^{-3}\right]$ is the concentration of iodine-135 at the instant $t$, $\gamma_{X e}$ is the fission yield of the nuclide xenon-135, $\lambda_{X e}$ given in $\left[h^{-1}\right]$ is the radioactive decay constant of xenon-135, $\gamma_{P m}$ is the fission yield of the nuclide promethium-149, $C_{P m}(t)$ given in $\left[\mathrm{cm}^{-3}\right]$ is the concentration of promethium-149 in time $t$ e $\lambda_{P m}$ given in $\left[h^{-1}\right]$ is the radioactive decay constant of promethium-149.

Considering the following initial conditions for the system of equations in (9): 


$$
\begin{aligned}
& n_{0}=1 \\
& C(0)=\frac{\beta}{\lambda \Lambda} n_{0} \\
& C_{I}(0)=\frac{\gamma_{I} \sum_{f} v}{\lambda_{I}} \\
& C_{X e}(0)=\frac{\gamma_{X e} \sum_{f} v+\lambda_{I} C_{I}(0)}{\lambda_{X e}+\sigma_{a, X e} v} . \\
& C_{P m}(0)=\frac{\gamma_{P m} \sum_{f} v}{\lambda_{P m}} \\
& C_{S m}(0)=\frac{\lambda_{P m} C_{P m}(0)}{\sigma_{a, S m} v}
\end{aligned}
$$

Similarly, we rewrite Equation (9) in its matrix form, where we separate the linear part from the nonlinear terms of the system

$$
\frac{d \mathbf{y}(t)}{d t}=\mathbf{M}(t) \mathbf{y}(t)+\mathbf{Q}(t) \mathbf{y}(t),
$$

where

$$
\begin{gathered}
\mathbf{y}(t)=\left(\begin{array}{c}
n(t) \\
C(t) \\
C_{I}(t) \\
C_{X e}(t) \\
C_{P m}(t) \\
C_{S m}(t)
\end{array}\right), \mathbf{M}(t)=\left(\begin{array}{ccccccc}
\frac{\rho-\beta}{\Lambda} & \lambda & 0 & 0 & 0 & 0 \\
\frac{\beta}{\Lambda} & -\lambda & 0 & 0 & 0 & 0 \\
\gamma_{I} \sum_{f} v & 0 & -\lambda_{I} & 0 & 0 & 0 \\
\gamma_{X e} \sum_{f} v & 0 & \lambda_{I} & -\lambda_{X e} & 0 & 0 \\
\gamma_{P m} \sum_{f} v & 0 & 0 & 0 & -\lambda_{P m} & 0 \\
& 0 & 0 & 0 & 0 & \lambda_{P m} & 0
\end{array}\right) \text { and } \\
\mathbf{Q}(t)=\left(\begin{array}{ccccccc}
0 & 0 & 0 & -\sigma_{a, X e} v n(t) & 0 & -\sigma_{a, S m} v n(t) \\
0 & 0 & 0 & 0 & 0 & 0 \\
0 & 0 & 0 & 0 & 0 & 0 \\
0 & 0 & 0 & -\sigma_{a, X e} v n(t) & 0 & 0 \\
0 & 0 & 0 & 0 & 0 & 0 \\
0 & 0 & 0 & 0 & 0 & -\sigma_{a, S m} v n(t)
\end{array}\right) .
\end{gathered}
$$




\subsection{Rosenbrock's Method}

Rosenbrock's methods belong to one of the classes of Runge-Kutta methods. Consist in linearizing the implicit Runge-Kutta methods, thus facilitating the implementation of the code, since instead of solving nonlinear systems, a sequence of linear systems is solved.

The solution of the systems in (5) and (9) at each time step is given by

$$
y\left(t_{0}+h\right)=y\left(t_{0}\right)+b_{1} k_{1}+b_{2} k_{2}+b_{3} k_{3}+b_{4} k_{4}
$$

where $t_{0}$ is the initial time, $h$ is the size of the integration step, $b_{i}{ }^{\prime} s$ are the method coefficients and the $k_{i}{ }^{\prime} s$ are the vectors corresponding to the stage of the method.

The structure of Rosenbrock's method adapted and used in this paper is found in Yang and Jevremovic [12], defined as:

$$
\begin{aligned}
& L k_{1}=f\left(y_{0}, t_{0}\right)+h c_{1} \frac{\partial f\left(y_{j}, t_{j}\right)}{\partial t} \\
& L k_{2}=f\left(y_{0}+a_{21} k_{1}, t_{0}+h\right)+\left[h c_{2} \frac{\partial f\left(y_{j}, t_{j}\right)}{\partial t}+\frac{c_{21} k_{1}}{h}\right] \\
& L k_{3}=f\left(y_{0}+a_{31} k_{1}+a_{32} k_{2}, t_{0}+h\right)+\left[h c_{3} \frac{\partial f\left(y_{j}, t_{j}\right)}{\partial t}+\frac{c_{31} k_{1}+c_{32} k_{2}}{h}\right], \\
& L k_{4}=f\left(y_{0}+a_{31} k_{1}+a_{32} k_{2}, t_{0}+h\right)+\left[h c_{4} \frac{\partial f\left(y_{j}, t_{j}\right)}{\partial t}+\frac{c_{41} k_{1}+c_{42} k_{2}+c_{43} k_{3}}{h}\right]
\end{aligned}
$$

where $L=\left[\frac{1}{\gamma h} I-\frac{\partial f\left(y_{j}, t_{j}\right)}{\partial t}\right], I$ is the identity matrix, $\gamma$ is one of the roots of the Laguerre polynomial and $\frac{\partial f\left(y_{j}, t_{j}\right)}{\partial y}$ is the Jacobian matrix, where $t_{j+1}=t_{j}+h$ and $y_{j}$ is the solution evaluated at $t=t_{j}$ for each $j$ in the mesh. The values of the other parameters of the method can be found in Yang and Jevremovic [12]. 


\section{RESULTS AND DISCUSSION}

In this section, the results are presented for the model with temperature feedback and, subsequently, for the model coupled to the effects of the main neutron absorbers poisons. The software used to implement the algorithm was SciLab 6.0.2 (http://www.scilab.org/).

\subsection{Temperature Feedback Model}

In this case of ramp reactivity insertion with Newtonian feedback, the kinetic parameters from Table 5 found in Tumelero [7] are used. We emphasize that the work of the reference [7] makes a broad systematic review of the main methodologies already used to solve the Point Kinetics equations over the years. Therefore, this paper compiles the main cases and core parameters to verify the proposed methodologies.

The initial conditions used are: $\rho_{0}=0$ and $n_{0}=1 \mathrm{~cm}^{-3}$. Four values are considered for reactivity, $\rho=0.1 t \$, \rho=0.01 t \$, \rho=0.003 t \$$ and $\rho=0.001 t \$$, and two values for the parameter $B, B=10^{-11}$ and $B=10^{-13}$. The " $t$ " is the time variable indicating that the reactivity is expressed in linear form. It is worth noting that reactivity is a dimensionless quantity that represents how far the system is from criticality, i.e., it is nothing more than a relative deviation. There are some units assigned to it, such as the Dollar (\$) that was used, which is equivalent to $\beta$.

The results obtained for the neutron density with their respective deviations are presented in Tables 1 and 2, considering the time step of $\Delta t=0.0001 / 2 s$.

The results are compared with the improved constant piecewise constant approximation (EPCA) method of Picca et al. [13]. The EPCA method [13] is considered a "benchmark" in reactor physics for its history and excellent contributions in the area. Therefore, we can consider them as good references for validation of the methodology. 
Table 1: Neutron density in $\mathrm{cm}^{-3}$ with feedback, for $B=10^{-11}$ and different cases of ramp-type

\begin{tabular}{ccccc}
\hline Reactivity & $t(s)$ & $\begin{array}{c}\text { ROS } \\
\Delta t=0.0001 / 2 s\end{array}$ & EPCA & $\begin{array}{c}\text { Relative } \\
\text { Deviations }\end{array}$ \\
\hline \multirow{5}{*}{$\rho=0.1 t \$$} & 0.1 & 24.7336582 & 24.7336582 & 0 \\
& 0.5 & $9.94989333 \mathrm{E}+09$ & $9.94989324 \mathrm{E}+09$ & $9.0453231837 \mathrm{E}-09$ \\
& 5 & $1.00297404 \mathrm{E}+10$ & $1.00297404 \mathrm{E}+10$ & 0 \\
& 7.5 & $1.00179842 \mathrm{E}+10$ & $1.00179842 \mathrm{E}+10$ & 0 \\
& 10 & $1.00118861 \mathrm{E}+10$ & $1.00118861 \mathrm{E}+10$ & 0 \\
\hline & 0.1 & 1.167210837 & 1.167210838 & $8.5674324418 \mathrm{E}-10$ \\
& 0.5 & 4.269952844 & 4.269952844 & 0 \\
\hline$=0.01 t \$$ & 5 & $1.033798290 \mathrm{E}+09$ & $1.033798290 \mathrm{E}+09$ & 0 \\
& 7.5 & $1.019490285 \mathrm{E}+09$ & $1.019490285 \mathrm{E}+09$ & 0 \\
\hline \multirow{5}{*}{$0.003 t \$$} & 10 & $1.012431671 \mathrm{E}+09$ & $1.012431671 \mathrm{E}+09$ & 0 \\
& 0.1 & 1.045371666 & 1.045371666 & $7.5490956283 \mathrm{E}-10$ \\
& 0.5 & 1.324661984 & 1.324661985 & 0 \\
& 5 & $3.29326626 \mathrm{E}+08$ & $3.293266270 \mathrm{E}+08$ & $3.0364990802 \mathrm{E}-09$ \\
& 7.5 & $3.22719218 \mathrm{E}+08$ & $3.227192182 \mathrm{E}+08$ & $6.1973377698 \mathrm{E}-10$ \\
& 10 & $3.14593641 \mathrm{E}+08$ & $3.145936416 \mathrm{E}+08$ & $1.9072222723 \mathrm{E}-09$ \\
\hline & 0.1 & 1.014717771 & 1.014717771 & 0 \\
& 0.5 & 1.089821393 & 1.089821394 & $9.1758154639 \mathrm{E}-10$ \\
& 5 & 14.16371139 & 14.16371139 & 0 \\
& 7.5 & $1.259267838 \mathrm{E}+09$ & $1.259267838 \mathrm{E}+09$ & 0 \\
& 10 & $1.59005860 \mathrm{E}+08$ & $1.590058602 \mathrm{E}+08$ & $1.2578152764 \mathrm{E}-09$ \\
\hline
\end{tabular}


Table 2: Neutron density in $\mathrm{cm}^{-3}$ with feedback, for $B=10^{-13}$ and different cases of ramp-type reactivity.

\begin{tabular}{ccccc}
\hline Reactivity & $t(s)$ & $\begin{array}{c}\text { ROS } \\
\Delta t=0.0001 / 2 s\end{array}$ & EPCA & $\begin{array}{c}\text { Relative } \\
\text { Deviations }\end{array}$ \\
\hline \multirow{5}{*}{$\rho=0.1 t \$$} & 0.1 & 24.73365825 & 24.73365830 & $2.0215367817 \mathrm{E}-09$ \\
& 0.5 & $1.543361792 \mathrm{E}+12$ & $1.543361754 \mathrm{E}+12$ & $2.4621576828 \mathrm{E}-08$ \\
& 5 & $1.002974092 \mathrm{E}+12$ & $1.002974092 \mathrm{E}+12$ & 0 \\
& 7.5 & $1.001798437 \mathrm{E}+12$ & $1.001798437 \mathrm{E}+12$ & 0 \\
& 10 & $1.001188620 \mathrm{E}+12$ & $1.001188621 \mathrm{E}+12$ & $9.9881279014 \mathrm{E}-10$ \\
\hline & 0.1 & 1.167210837 & 1.167210838 & $8.5674324418 \mathrm{E}-10$ \\
& 0.5 & 4.269952864 & 4.269952865 & $2.3419462266 \mathrm{E}-10$ \\
& 5 & $1.033889665 \mathrm{E}+11$ & $1.033889665 \mathrm{E}+11$ & 0 \\
& 7.5 & $1.019499912 \mathrm{E}+11$ & $1.019499913 \mathrm{E}+11$ & $9.8087306065 \mathrm{E}-10$ \\
& 10 & $1.012434883 \mathrm{E}+11$ & $1.012434883 \mathrm{E}+11$ & 0 \\
\hline \multirow{5}{*}{$0.01 t \$=0.003 t \$$} & 0.1 & 1.045371666 & 1.045371667 & $9.5659757344 \mathrm{E}-10$ \\
& 0.5 & 1.324661986 & 1.324661986 & 0 \\
& 5 & $3.215676113 \mathrm{E}+10$ & $3.215676113 \mathrm{E}+10$ & 0 \\
& 7.5 & $3.210205182 \mathrm{E}+10$ & $3.210205182 \mathrm{E}+10$ & 0 \\
& 10 & $3.145614686 \mathrm{E}+10$ & $3.145614687 \mathrm{E}+10$ & $3.1790289005 \mathrm{E}-10$ \\
\hline & 0.1 & 1.014717771 & 1.014717771 & 0 \\
& 0.5 & 1.089821394 & 1.089821395 & $9.1758154555 \mathrm{E}-10$ \\
& 5 & 14.16371386 & 14.16371386 & 0 \\
& 7.5 & $8.361617706 \mathrm{E}+09$ & $8.361617606 \mathrm{E}+09$ & $1.1959408419 \mathrm{E}-09$ \\
& 10 & $1.615876766 \mathrm{E}+10$ & $1.615876767 \mathrm{E}+10$ & $6.1885907417 \mathrm{E}-10$ \\
\hline
\end{tabular}

Tables 1 and 2 show that the results obtained are excellent when compared to the EPCA method, with the largest relative deviation of $2.4621576828 \mathrm{E}-08$. In general, in most cases, there is an approximation of all decimal places when comparing the results to the EPCA method.

The EPCA method is a semi-analytical method based on a piecewise constant approximation. It consists in finding the solution iteratively in each time step, considering a sub-mesh to improve the accuracy of the numerical quadratures. In this way, the solutions found are highly accurate. Rosenbrock's method, on the other hand, provides an L-stable numerical solution (ideal for stiff problems) and uses only one Newton iteration per integration step, and can achieve both accuracy and stability. Note that these are different approaches, but they achieve several digits of accuracy.

In this sense, it is noted that Rosenbrock's method produced excellent results when compared with those in the literature by solving a nonlinear problem when considering temperature effects 
and also with time dependence in reactivity. Furthermore, the results obtained served to verify the methodology proposed in this work.

\subsection{The Model with the Effects of Neutron Absorbers Poisons}

In the first simulation case considering the effects of poisons, a low-power reactor with negative reactivity was considered, $\rho=-0.0001$, generated by $100 h$ for neutron density, delayed neutron precursor concentration, and for the concentration of xenon-135. For the concentration of samarium-149, the graph was generated until $500 h$. It is emphasized that the same approach of Espinosa [8] was followed, in which reactivity is decomposed into short and long scale contributions. The first represents the operational control of the reactor, while the second occurs due to the variation of the chemical composition of the nuclear fuel as a consequence of decay.

The following kinetic parameters were used: $\beta=0.0065, \lambda=278.68 h^{-1}$ and $\Lambda=2.7778 \times 10^{-8} h$ with initial conditions and nuclear parameters found in Paganim et al. [9].

As previously mentioned, there are not many works in the literature that take into account the effects of poisons on NPKEs, at least not to the authors' knowledge. In this sense, the kinetic parameters used in [9] have physical plausibility with the simulation proposed in this work.

The plots for neutron density, delayed neutron precursor concentration, xenon-135 and samarium-149 concentration are shown in Figure 1. It is observed from Figure 1 items (a) and (b) that neutron density and delayed neutron precursor concentration have similar behavior. Both decay after a while, due to negative reactivity, causing the reactor to shutdown, tangenting the abscissa axis around $t=5 \mathrm{~h}$ and reaching the absolute value of zero at $t=11 \mathrm{~h}$.

When the neutron density begins to tangent the abscissa axis, that is, its value is practically zero mathematically, physically the reactor is shutdown. It can be interpreted that a residue occurs between the mathematical solution and the one that physically occurs due to the exponential decay of the solution. After the reactor is shut down (when mathematically the solution approaches zero at about $5 h$ ), the concentration of Xe-135 continues to increase until it reaches its peak at about ten hours, due to the absence of xenon-135 consumption by radiative capture and production through iodine-135 decay. However, it is observed that the concentration of xenon-135 remains in the reactor for up to eighty hours, about three days, decreasing its concentration by the reduction of 
iodine-135 and by its decay. A resumption of power during this period of contamination would require more neutrons to compensate for the negative reactivity caused by the poisoning. Therefore, it is important to wait at least three days for the safe restart of a nuclear reactor after a shutdown.

The samarium-149 (item (d)), unlike xenon-135, grows up to a certain value and stabilizes after a while, approaching a constant value in its concentration during the operation of the reactor until the complete decay of promethium-149. This happens because the element samarium-149 is stable through its decay chain, and the constant value that will be reached depends on the previous power of the reactor.

In the second simulation case, we used as initial condition the results at instant $30 \mathrm{~h}$ of the first case. The simulation consists of restarting the reactor before the xenon-135 is consumed. For this purpose, a positive reactivity was inserted, $\rho=0.01 \beta$ to capture the effects of neutron absorbers poisons on the resumption of power in an already contaminated reactor.

The results of the behavior of neutron density, precursor concentration, iodine-135 concentration, xenon-135 concentration, promethium-149 concentration and the element samarium149 are presented in Figure 2. 
(a)

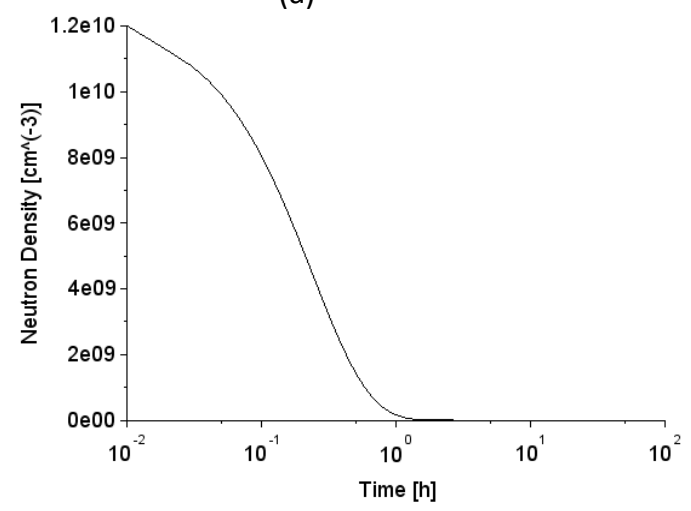

(c)

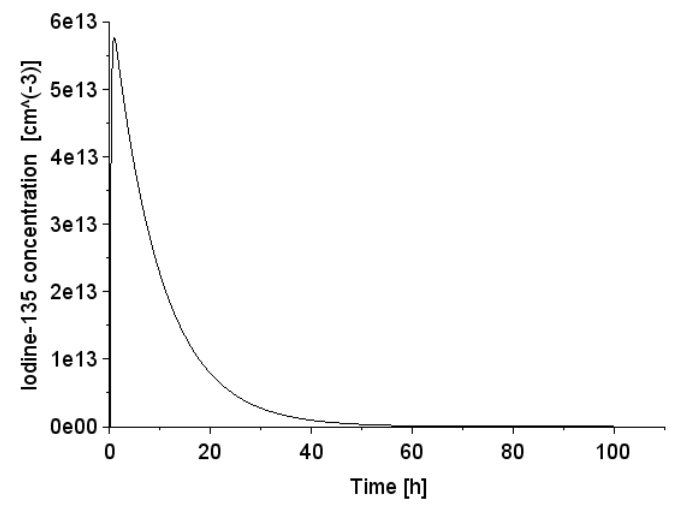

(b)

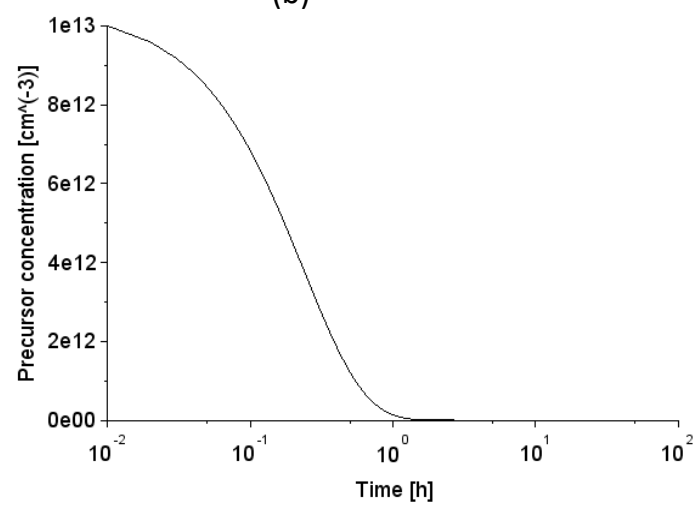

(d)

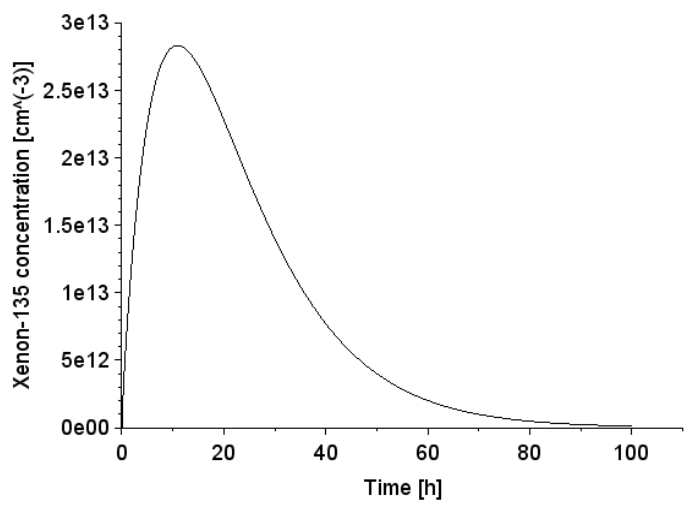

(e)

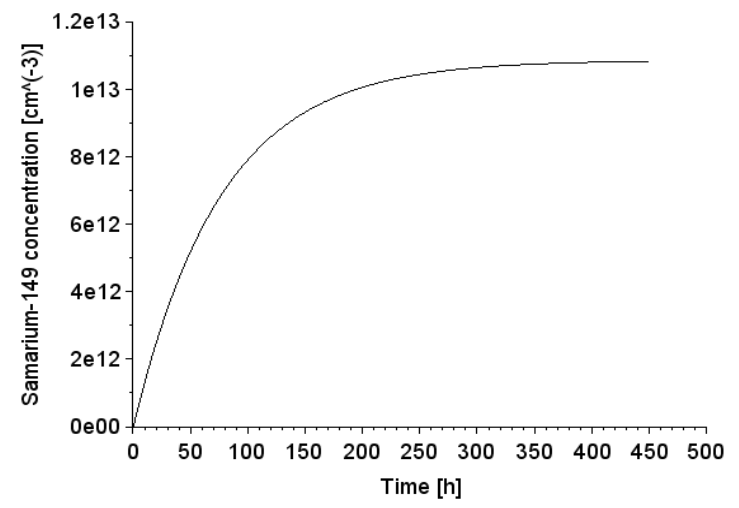

Figure 1: Graphics for reactivity $\rho=-0.0001$, (a) Neutron Density, (b) Precursor concentration, (c) iodine-135 concentration, (d) xenon-135 concentration and (e) samarium-149 concentration. 
(a)

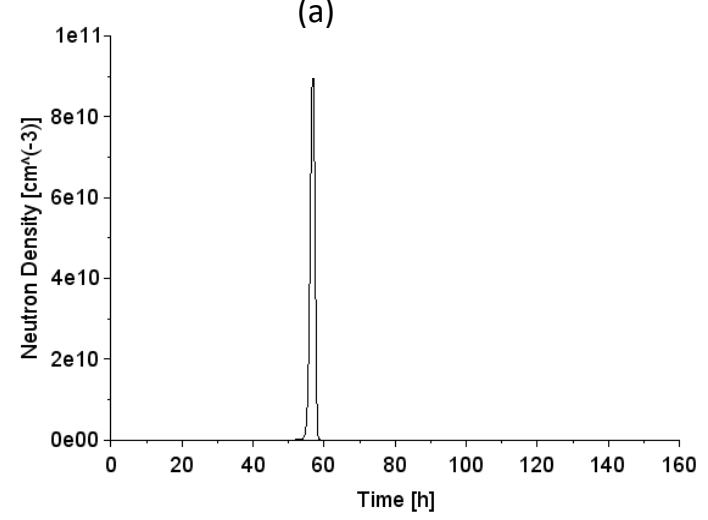

(c)

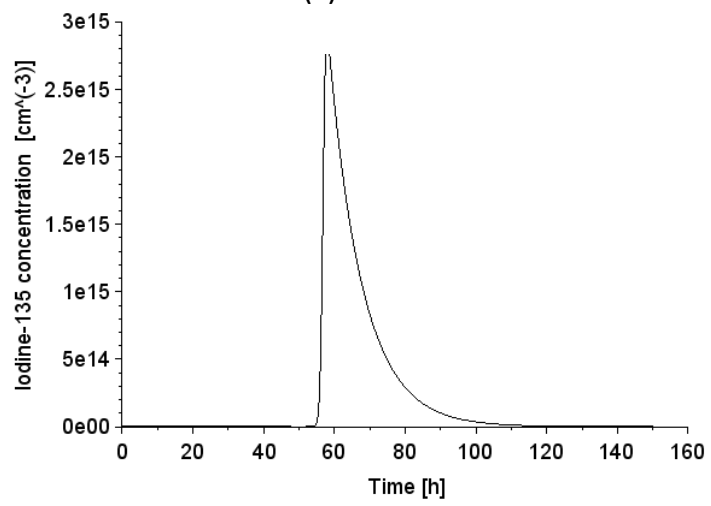

(e)

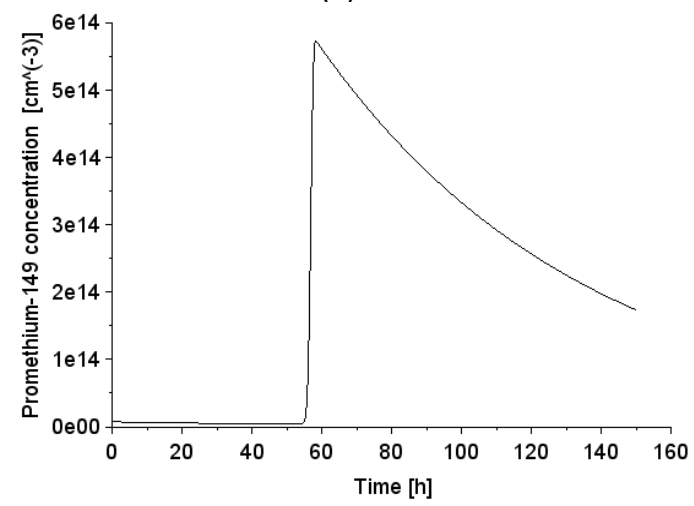

(b)

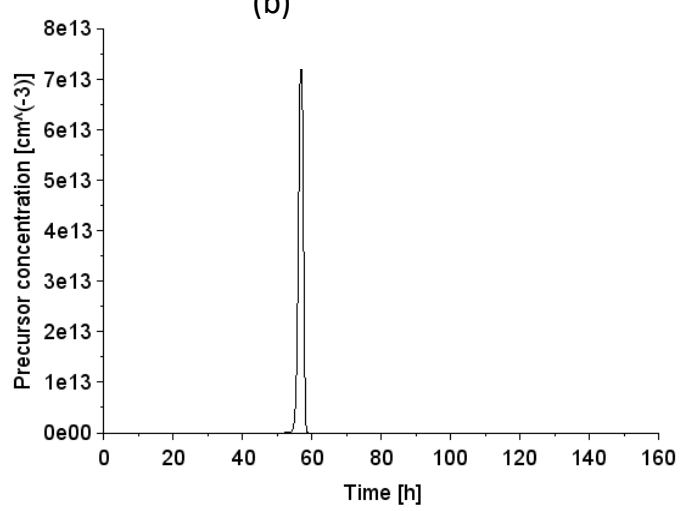

(d)

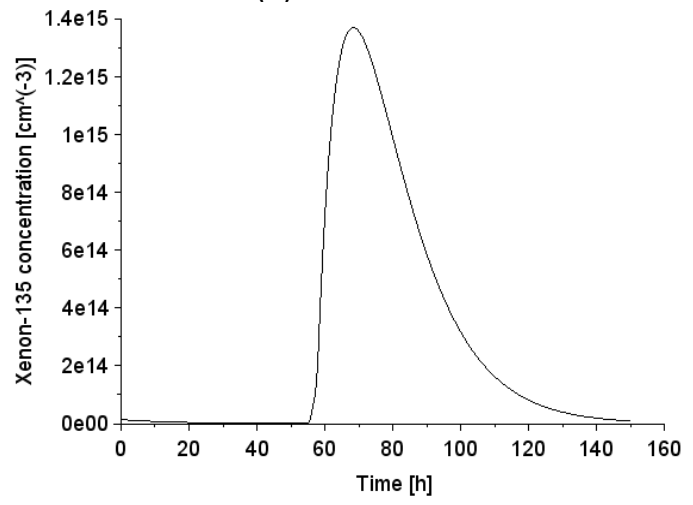

(f)

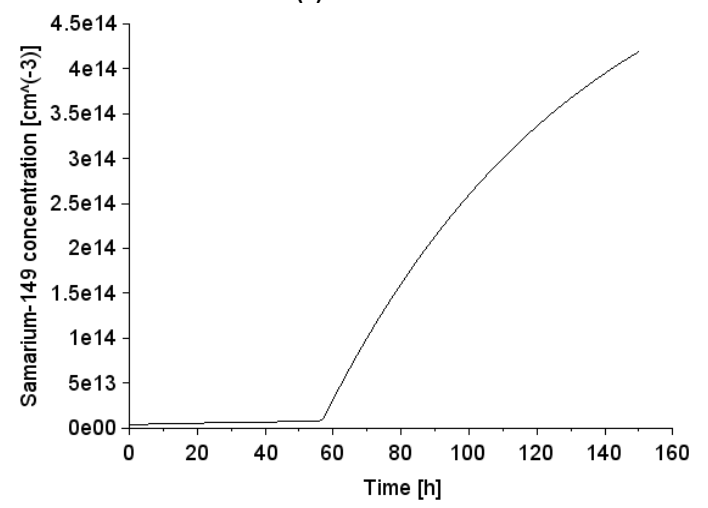

Figure 2: Graphics for reactivity $\rho=0.01 \beta$, (a) Neutron Density, (b) Precursor concentration, (c) iodine-135 concentration, (d) xenon-135 concentration, (e) promethium-149 concentration and (f) samarium-149 concentration. 
The initial conditions imposed on the reactor describe a system already contaminated by the neutron absorbers poisons, where the reactor was already shutdown.

It can be seen from the graphs shown in Figure 2 that the positive reactivity caused an increase in neutron density and precursor concentration, peaking near $60 h$, as shown in Figure 2 (a) and in the delayed neutron precursor concentration, Figure 2(b). This increase consequently raised the number of fissions and, in turn, the production of the element promethium-149 (Figure 2 (e)), which peaked at approximately $60 \mathrm{~h}$. As a result, the nuclide samarium-149 (Figure 2 (f)) began to grow sharply, capturing neutrons and causing the density value to decrease. It is also possible to observe that neutron capture poisoning occurs from xenon-135 (Figure 2 (d)), which is produced by fission (before shutdown) and decay of iodine-135 (Figure 2 (c)). When the reactor shuts down, the xenon135 continues to grow because radiative capture no longer occurs and is fueled by the decay of iodine-135, peaking at approximately $70 \mathrm{~h}$.

We emphasize that there is no restriction to generate results by increasing the time interval. In fact, the idea in the future is to simulate the effects of the main neutron absorbers poisons in the Chernobyl accident, which had its significant increase after $10 \mathrm{~h}$ of the reactor operating at half the designed power.

\section{CONCLUSIONS}

In this paper, the solution of the Neutron Point Kinetics equations coupled to temperature effects with ramp-type reactivity for six groups of delayed neutron precursors by the Rosenbrock's method was presented. We also solved the Point Kinetics model considering the effects of the main neutron absorbers poisons for one precursor group and constant reactivity.

The choice of an L-stable method was to guarantee future solutions for stiff problems, considering simulations for large amounts of hours. Moreover, as it is intended to simulate the Chernobyl accident, considering the effects, not only of the main neutron absorbers poisons, but also of the influence of temperature and more precursor groups, a methodology capable of providing accurate, stable and timely manner results were sought. 
In general, the ease of implementation of the algorithm, the possibility of using adaptive timestepping since it is a single-step method, in addition to the good results in the literature when solving stiff problems, made Rosenbrock's method the choice for the solution of the models proposed in this work.

The results presented for the model with temperature effects were excellent when compared to the literature. The stiffness of the system of ordinary differential equations was overcome by the implicit formulation of the method, emphasizing that these are also nonlinear problems.

It is worth mentioning that the results obtained served to verify the methodology proposed in this work, making it feasible to solve the model coupled to the effects of the main neutron absorbers poisons.

Therefore, it was proposed, after the verification of the methodology, to solve the Point Kinetic model coupled to the effects of the fission products poisons. The importance of this study comes to meet the need to perform a shutdown for recharge or maintenance of a nuclear reactor, where the importance of waiting for the decay time of the neutron absorbers poisons is highlighted for a safe and efficient resumption of power. In the literature, there are works done in this sense, but simulations have been presented that were previously unpublished in the literature, exemplifying this situation. Furthermore, the use of the Rosenbrock's method to solve this problem, considering the neutron absorbers poisons, stands out as a novelty.

It was found that the methodology achieved its goal by simulating the behavior of fission product poisons in such a way that the graphs have a physical meaning consistent with what is expected from ordinary differential equations.

Fission product poisons play an extremely important role in balancing the neutron flux, in that they absorb neutrons, slowing the reactor response time due to this insertion of negative reactivity. The neglect of these effects of the poisoning at Chernobyl resulted in test procedures and safety regulations being broken in order to resume power to perform a test. After this, numerous system problems are detected that contribute to a completely unstable reactor leading to a thermal core explosion.

Therefore, Rosenbrock's method obtained a remarkable performance in solving nonlinear problems, bypassing the degree of stiffness of the system of equations, making it feasible to use the 
methodology for other cases of simulations, important for safety and decision making in reactor physics.

As prospects, it is intended to expand the simulation with the effects of absorbers poisons, bringing a context of the Chernobyl disaster, from accident data present in the literature. Specifically, it is intended to simulate a power resumption after the decay time of the fission product poisons, to analyze the reactor response time in this scenario.

\section{ACKNOWLEDGMENTS}

The first author thanks the Coordenação de Aperfeiçoamento de Pessoal de Nivel Superior (CAPES) for the financial incentive to this research. The other authors thank the Federal University of Pelotas (UFPel) for the support for this work.

\section{REFERENCES}

[1] DUDERStADT, J. J.; HAMILTON, L. J. Nuclear Reactor Analysis, John Wiley \& Sons, New York, 1976.

[2] LAMARSH, J. R. Introduction to Nuclear Reactor Theory, New York: Wesley publishing company, 1966.

[3] ZANDONÁ, J. C.; SCHAUN, N. B.; PETERSEN, C. Z.; TUMELERO, F.; SCHRAMM. Solução das equações da cinética pontual de nêutrons via Método de Fator de Integração Implícito com fonte externa. Braz. J. Radiat. Sci, v. 8, p. 1-18, 2020.

[4] SILVA, J. J. A. Cinética Pontual com Realimentação de Temperatura considerando um grupo de precursores de nêutrons atrasados. Dissertação de Mestrado — UFRGS, Porto Alegre/RS, 2011.

[5] TASHAKOR, S.; JAHANFARNIA, G.; E HASHEMI-TILEHNOEE, M. Numerical solution of the point reactor kinetics equations with fuel burn-up and temperature feedback. Ann. Nucl. Energy, 3378, v. 37, p. 265-269, 2011. 
[6] PETERSEN, C. Z.; DULlA, S.; VILHENA, M. T. B.; RAVETTO, P. An analytical solution of the point kinetics equations with time-variable reactivity by the decomposition method. Prog. Nucl. Energy, v. 53, p. 1091-1094, 2011.

[7] TUMELERO, F. Solução das Equações da Cinética Pontual de Nêutrons com e sem Retrolimentação de Temperatura pelo Método da Aproximação Polinomial. Dissertação de Mestrado - PPGMMat/UFPel, Pelotas, 2015.

[8] ESPINOSA, C. E. Modelagem e Simulação dos venenos no combustível nuclear em cenário com duas escalas de tempo. Tese de Doutorado - UFRGS, Porto Alegre/RS, 2016.

[9] PAGANIN, T.; BODMANN, B.; VILHENA, M. On a point kinetic model for nuclear reactors considering the variation in fuel composition. Prog. Nucl. Energy, 0149-1970, v. 118, p. 103$134,2020$.

[10] GALINA, G. Sistemas Rígidos Associados a Cadeias de Decaimento Radioativo. Dissertação de Mestrado - IME-USP, São Paulo, 2016.

[11] SEHNEM, R. Modelagem numérica para a obtenção de mecanismos reduzidos via método de Rosenbrock: a combustão do metano. Dissertação de Mestrado PPGMMat/UFPel, Pelotas, 2018.

[12] YANG, X.; JEVREMOVIC, T. Revisiting the Rosenbrock numerical solutions of the reactor point kinetics equation with numerous examples. Nucl. Technol. Radiat. Prot, v. 24, p. 3-12, 2009.

[13] PICCA, P.; FURFARO, R.; GANAPOL, B. A. highly accurate technique for the solution of the non-linear point kinetics equations. Ann. Nucl. Energy, 0306-4549, v. 58, p. 43-53, 2013. 